\title{
Laboring Artists: Art Streaming on the Videogame Platform Twitch
}

\author{
Andrew M. Phelps \\ University of Canterbury, HITLabNZ (and) \\ American University, School of Communication \\ Christchurch, NZ, and Washington, DC, USA \\ andymphelps@gmail.com
}

\begin{abstract}
The relationship between labor and play is complex and multifaceted, particularly so as it relates to the playing of games. With the rise of the online streaming of games and play these platforms and activities have expanded the associated practices in ways that are highly nuanced and dictated in part by the platform itself. This paper explores the question as to whether the types of labor practices found in games hold across other non-game activities as they engage with streaming through an observational study of art streamers on Twitch. By examining art streamers and comparing their labor to that of games and game streaming, we find that not only are they similar in practice, but that that the structure of Twitch and platforms such as YouTube push this conformity. Thus, play and labor are not opposed and are in fact intermingled in these activities, in ways that are becoming highly platformized.
\end{abstract}

\section{Introduction}

Although we often talk about "playing a game," labor is intertwined with gameplay in myriad ways. This applies to practices we enact while playing games, including gold-farming, grinding, powerleveling, creation of unofficial guides and theorycrafting, as well as to the streaming of gameplay and the creation and uploading of pre-recorded gameplay videos, all of which can require increasingly complex forms of labor - whether for profit or not.

Such practices do not appear out of nowhere, and specific strategies and actions have emerged over time, often in relation to the platform or genre in which gameplay takes place. Yet even as games scholars have pointed to this complex history of "game labor," little account has been taken to examine how a particular platform on which a game appears, or a game-as-platform, can influence the types of labor that takes hold. We argue that the types of labor practices found in games, and the streaming of games, is increasingly reliant on commercial structures such as

\author{
Mia Consalvo \\ Concordia University \\ Department of Communication Studies \\ Montreal, Canada \\ mia.consalvo@concordia.ca
}

Twitch and YouTube, which push an increasing conformity for laboring individuals that is spilling over into other types of (non-gaming) streaming activities. To justify this assertion, this paper does two things: it revisits and highlights key findings from past game studies research that has examined game related labor practices and suggests how platforms played a role in shaping labor practices; and via a case study of art/game streamers it demonstrates how individuals engage in play, community building, art creation and self-promotion of their work via sites such as Twitch which provide a new layer of "authenticity" to their labor, with numerous parallels to the myriad of ways games and labor are interwoven. It draws comparisons between work on games and labor and emerging practices in art streaming, as streamers focus beyond games as the core subject matter, and illustrates how streaming is both an activity and a platform for these conditions. This paper's research question is: how do (game) platforms encourage particular forms of player-related labor, and how is that labor reappearing in non-game spaces? To answer that question, we rely on political economy theories of games and labor and engage in a digital ethnography of live streamed art-creation channels on Twitch.

\section{Labor and play, from games to streams}

In relation to digital games, political economy theorists Dyer-Witheford and de Peuter posit most labor today is actually "immaterial labor" or "work that creates 'immaterial products' such as 'knowledge, information, communication, a relationship or an emotional response" [17]. Similarly Boellstorff argues we are now seeing the spread of "creationist capitalism" where "labor is understood in terms of creativity, so that production is understood as creation" [6].

But even as platforms are seen as essential nodes for understanding such practices (such as the differing coding requirements for an XBOX or Twine game), game studies has not often seen platforms as formative in pushing player labor in particular directions. In their 
introduction to the Platform Studies series at MIT Press, Bogost and Montfort write platforms can be either software or hardware that forms the "underlying computing systems" that "enable, constrain, shape and support the creative work that is done on them." Taking a more political stance, Gillespie argues that the term "does not drop from the sky" but rather comes from "stakeholders with specific aims" [18]. We argue that platforms are indeed not neutral, and position Twitch as a platform worth investigating in this context.

The same forms of interactions that characterize games are emerging in art streaming on Twitch, as streamers engage in a range of activities that span from casual streams engaged in educational practice to commissioned work created live online replete with advertising, marketing, and more. We theorize that it is the platform itself that provides both the capabilities for this range of activities and the nudges to provide connection and adherence across them, and that existing work in games and media is thus relevant to understanding these emerging phenomena in additional 'game adjacent' spaces.

\section{Labor and playbour in games}

Game studies scholars often begin with theories of games and play from the pre-digital era. Drawing from Johan Huizinga, Roger Caillois, and Ludwig Wittgenstein among others, researchers conceptualized videogame play as the opposite of work, a voluntary activity, and something leisurebased $[23,26]$. Yet from the beginning, cracks appeared in these formulations of play as something disconnected from labor, work, or monetary concerns. One of the earliest challenges was Julian Kücklich's 2005 exploration of the role of modders in the larger games' ecosystem, and how their activities of modding resulted in complex relationships with game studios [24]. Kücklich deemed their activities 'playbour' in order to account for both the seriousness of the activity - the labor - as well as the play that initially drove such efforts. Kücklich pointed out that such activity was precarious at best given legal concerns over who owned mods, but also that modding was a key window into new forms of digital activity, or new ways of understanding how work and play were becoming intertwined. As he concluded about the entertainment industries more generally, "the relationship between work and play is changing, leading, as it were, to a hybrid form of "playbour."

\subsection{Modding}

Other researchers have studied modders and modding and have likewise demonstrated how the activity serves multiple ends: individuals can envision it as a creative practice or space, as a more-to-less serious hobby or leisure activity, and sometimes as a training ground for those with aspirations to work in the professional game industry $[31,34,35]$. But while all scholars have found that modders see what they do as work or labor to varying extents, they - and their respondents - dispute the charge that monetary compensation should always follow from their activities.

The role of platforms is also important to understanding modding. Many early games had no systematized file structure, and copy protection was not always employed, meaning players could poke around in a game's files, but usually couldn't figure out which parts actually did what. Yet with the growing standardization of file organization, players were able to start modifying files to do things like alter the images of characters or locations, and create new 'maps' on which to play [40]. As they became more inventive, modders began creating tools for even more ambitious changes - thus creating their own platforms for work [11]. Sites such as RomHacking.Net emerged to centralize and organize such efforts. Depending on the level of changes being attempted, early modders' efforts were often tolerated but sometimes legally challenged. Yet slowly developers recognized the value of modding - at least certain approved kinds of modding- and created official tools and platforms for modders to engage with. Yet those official platforms limited modding in key ways - offering only particular types of images or game assets, containing language about what were acceptable uses, declining modders' copyright of their work, and limiting the potential for monetization of those efforts. While certain popular games such as Minecraft still thrive without official modding platforms, the majority of such efforts have shaped what most potential modders see as possible forms of creative labor.

\subsection{Walkthroughs}

Modding is not the only form of game-related labor which players have participated in, of course, and been shaped by particular platforms. For example, players often create guides or walkthroughs of games they particularly enjoy, mainly to help other players get through puzzles, battles, and choices in the games. Consalvo [9] investigated player-created walkthroughs of Zelda 64 as a way to understand how players can also (re)produce narratives for games. For those who do so, that activity often comprises websites that are "dozens of pages in length, with minute levels 
of detail included" [9 p. 328]. She also points out that "the level of work involved and the dedication to the activity, which is usually not paid, can be tremendous" [9 p. 329]. While such early efforts were often illustrated text guides, the ability to freely upload walkthroughs, guides, and later "Let's Play" videos on sites such as YouTube quickly became the dominant way to share. However, as games have evolved into 'services' that constantly change, individuals creating video guides for them are now pushed to constantly update their offerings. As Consalvo also found, for such creators to retain credibility, they not only need expert knowledge of a game (such as Clash Royale), but must regularly post new, short, highly polished explainer videos about the game to keep their 'views' high [12].

Yet the demand for more knowledge about how to play a game, or play it optimally, is not always to all players' benefit. For example, some of the power gamers that Taylor studied demanded such high levels of play from their peers in World of Warcraft that they ultimately created mods such as CT Raid Assist to surveil and assess users, and certain guilds made them mandatory for members [37]. Here we see players working to create systems that judge one another, adding a new layer to the game's platform that was not originally present. Yet because Blizzard allowed for such modding (but always keeping a close eye and removing mods they deemed problematic), WoW as a platform supported such work, letting players expand the range of possibilities for how they could interact with each other in the game.

\subsection{Gold farming}

Game labor started to become more institutionalized and we witnessed more commercial structures supporting it with the popularization of selling in-game items on sites such as eBay and the rise of gold farming as a lucrative activity within MMOs. These activities either capitalized on existing sites or created new ones that both brought in-game items outside the game space (such as via eBay), and then decoupled labor from the work of an individual to a larger corporate entity through the gold selling site IGE.

Gold farming originally referred to an automated task rather than one engaged in by people, using the creation of in-game macros to automatically change "an input into a more valuable output" in order "to achieve financial gain" [25 p. 2730].

Eventually though bots to "farm" game objects were supplanted, as "most of the farming is done by real people in low-wage countries from Romania to China. 'Gold farming' is now a significant export that's creating new economic opportunities for young people in remote villages that have few other employers" [ 2 p. 733]. In contrast Warner and Raiter [39] argued that Chinese 'gold farms' "bring a new dimension to issues of inequity - through the economic implications of cross-over between real and virtual worlds, and through ethical questions regarding the disparate nature of relatively wealthy individuals in one culture paying a pittance for services performed by relatively poor individuals working in sweatshop conditions" [39 p. 50].

Attention to the individuals engaged in gold farming perhaps reached its peak in 2007 when Julian Dibbell traveled to China to meet gold farmers. As he explained, "while the Internet has produced some strange new job descriptions over the years, it is hard to think of any more surreal than that of the Chinese gold farmer" [16 n.p.]. But as Dibbell remarks, although the title sounds bizarre, "there is little to distinguish gold farming from toy production or textile manufacture or any of the other industries that have mushroomed across China to feed the desires of the Western consumer" [16 n.p.]. What is really only notable is that this happened within videogames and was therefore one of the earliest forms of precarious digital labor to emerge.

Yet the primary frame through which game studies engaged with gold farming was as a form of cheating [10]. The activity had been deemed illegal according to the game publishers that owned the MMOs where gold farming occurred, which fed into a larger system of "real-money trade." RMT, it was argued, let some players 'cheat' by paying with real currency for items or even virtual currency within such games rather than spend time in game to acquire such things themselves.

Other researchers drew attention to the individual farmers, and how their Asian bodies -invisible behind avatars - were subjected to racist discourses. Nick Yee argued that player discussions about and comments directed towards gold farmers have undeniable links to past racist imagery, and "the contemporary narrative starts to feel too much like the historical one - Chinese immigrant workers being harassed and murdered by Westerners who feel they alone can arbitrate what constitutes acceptable labor" [42 n.p.].

Lisa Nakamura extended his critique, arguing that such a system resulted in "Asian worker players [that] are economically unable to accumulate avatarial capital and thus become "persons;" they are the dispossessed subjects of synthetic worlds. As long as Asian "farmers" are figured as unwanted guest workers within the culture of MMOs, user-produced extensions of MMO-space like machinima will most likely continue to depict Asian culture as threatening" [28 p. 142]. 
The fact that the labor system of gold farming is both raced and classed lends further weight to the contention that these game-related activities prefigure in unsettling ways the precarious, contingent state of future digital economy workers. As Dibbell wrote about human gold farmers in China that he interviewed, "Twelve hours a night, seven nights a week, with only two or three nights off per month, this is what Li does - for a living" [16 n.p.]. Contrast that statement with an account of game live streamer Joe Marino: "I went for around 8 hours every day. That was just the live portion of my day. Next, I was on the phone with companies ... working out deals for sponsorship ...Repeat this 7 days a week" [27 n.p.]. Marino eventually gave up full time streaming because his health had declined so markedly from lack of exercise and work-related stress that he almost died. Yet others continue to work long hours streaming and a manta among Twitch partners is that in order to be profitable they must "always be streaming" [22].

\subsection{YouTube and Twitch}

YouTube and Twitch have also incentivized new forms of labor relative to gameplay that place the risk on the individual 'content creators' but with benefits accruing mainly to the companies themselves. On YouTube, the establishment of game commentary videos has brought millions of dollars and garnered celebrity for select individuals such as PewDiePie and Markiplier, but also demonstrates yet another way that the act of playing a game can be commodified and sold to an online audience, and also how platforms shape creators' efforts, whether they profit from them financially or not. This system further entrenches an expectation that monetization or the "optimization" of engagement should be expected parts of cultural production. Postigo provides an excellent analysis of how such YouTubers convert play into "making gameplay" and finally "making game pay" in a laborfilled process that is constant and without boundaries [32]. Postigo also points out that play itself is not the only component of such videos, but that "YouTubeworthy gameplay involves not only talent, but also the use of a number of technological and social structures that convey competitive advantage" [24, p. 11]. And beyond this work done by individuals who can be more and less successful at it, all such work is ultimately in service of YouTube itself, because "should a channel shrink or a genre go out of fashion, another will take its place and YouTube's architecture will accommodate it and gets its share of cash" [24, p. 14].

The labor and business logics established by YouTube have also been carried over into the practice of live streaming of gameplay, and further refined by companies such as Twitch.tv. Twitch itself has followed a curious path, starting as a life-streaming platform, narrowing to videogame streaming in 2011, and most recently allowing for 'In Real Life' (IRL) channels to exist (again), as well as a variety of channels focusing on creative activities such as art making, videogame development, crafting, cooking, and many others. Yet Twitch is known primarily as a space for streaming yourself playing videogames, and the logics of how Twitch promotes the monetization of game streams (via gamification of its pathways to affiliate and partner, for example) unavoidably spill over between - just as the audiences too move between - the game and non-game content on the platform.

Despite it being a site where the vast majority of streamers make no revenue from doing so, the majority of research on Twitch has focused on large and successful streamers, particularly those engaged in eSports and competitive multiplayer games $[15,20,22,38]$. Scholars have pointed to the extensive labor involved in creating a Twitch setup (PC, specialized software, webcams, greenscreens, microphones and often more) as well as the work involved in maintaining a "persona" while streaming, or at the least being entertaining and interacting with potential viewers, often for many hours at a stretch $[8,22,38]$. Different types of investigations of game streamers have also begun to emerge, but because Twitch bills itself as a way for individuals to make money while doing something they ostensibly enjoy, this framing was perhaps inevitable.

Yet as we see more nuanced attention to game live streaming, researchers have also begun to investigate play-adjacent practices on Twitch. For example, Consalvo and Phelps have studied game developers who live stream themselves building games [13], and Wohn has interviewed moderators for streamers to better understand their motivations and beliefs about the job [41]. Yet even as we move away from a focus on games or play itself, the centrality of labor to the process - in various forms - is undeniable, if even in a playful sense.

\section{Art streaming}

Art streaming, or art development streaming, is an underexplored area of Twitch that shares many facets of its operation with other hobby- or quasiprofessional-based streaming communities. At its core, art streaming is the activity of streaming the creation of art: drawing, painting, sculpture, etc, as well as their digital counterparts such as digital illustration or 3D modelling. The terminology of 'art' 
in this context is primarily limited to the traditional definition of the fine arts, as opposed to other activities that are both streamed and could be considered 'art' in a broader context such as game design or development, music, and several crafts and hobbies. This was in fact specifically platformized itself as a dedicated channel on Twitch, the so-called 'Art Channel' which is in the 'Creative' section of Twitch, and which employs this fine arts definition for said content. Interestingly, there is substantial consternation within this community that the deletion of 'Twitch IRL' and subsequent creation of specialized channels by Twitch itself within the 'Creative' umbrella [1] has damaged the overall general audience of viewers, as it has discouraged those outside the activity to browse the content in an integrated dashboard.

To better understand the practices and motivations of art streamers, the authors have undertaken an analysis of this community. This paper explores early observations over approximately a year, during which different streams were selected several times weekly from the first three pages of the 'Art' channel, with an effort to vary the gender, presumed age, location, and artistic subject of the streamer. Streams were observed for an hour or more, both watching the stream live and monitoring the chat in real-time. Field notes were taken on the activities and conversations therein and analyzed later to draw conclusions and recognize repeated patterns of behavior. Given that these streams were all publicly available, and that neither the streamers nor their audience members were interviewed or interacted with directly, IRB approval for this observation was deemed inapplicable. As such, this paper represents early work in exploring and understanding these activities: a more formalized methodology and ethnography is planned for further study. Approximately 280 different streamers have been observed, some of them repeatedly. It should be noted that the vast majority of these streams are quite small, with typical audience sizes of less than 5 and often 0 or 1 (subtracting out the authors observation). Furthermore, this study only selected streams focused on various forms of 2-dimensional drawing and painting, including traditional media (watercolor, acrylics, oils, pencil, pastels, and mixed media) as well as digital media.

\subsection{Motivations and platform interactions}

The motivations of art streamers are varied, but several themes re-occur during both chat discussions and observed practice that bear strong similarity to the prior discussion on games, play, and labor. First is the engagement in the work of the activity itself. Past research on Twitch streamers who engage in game development found that one of the stated motivations of such streamers is to force themselves into a daily practice [13] - i.e. that streaming motivates a daily commitment via the platform. This overlaps standard art education practices that often rely on daily sketching, painting, or drawing as a form of immersion and practice of technique and craft and is often assigned in introductory courses in studio disciplines. In observation, a number of streamers also identified that their primary motivation for engaging in the activity is simply to 'learn to make better art' through a combination of streaming their own practice and getting feedback (see below), being able to review their own process by recording their stream, by watching the streams of others for new techniques and ideas, and by seeking to engage in a community of practitioners that, to some extent, mimic a more traditional studio culture. Needless to say, this activity is obviously and foremost a form of individual labor, albeit purposefully and directly engaged in by choice as the purpose of engaging in art streaming is to engage in the creation of art.

It must also be noted that this activity is also a form of negotiated labor in terms of engagement with the platform: how to best stream art creation is itself an open question, just as Taylor found with respect to variety streamers who were constantly tweaking and adjusting their streaming protocols [38]. Perhaps unsurprisingly, many art streamers attempt to mimic a formulaic step-wise, talk aloud protocol as popularized most famously by Bob Ross on his television program "The Joy of Painting" [44], but there are numerous exceptions and experiments. Indeed, the retro-style Ross stream [45] is the most widely viewed in this entire sub-culture, often with a focus on merely connecting socially while satirizing the medium (and in particular speculate on "cabin chance" [46] during the creation of Ross' work, meaning the chance that a given painting during an episode of the show will contain a cabin as a part of the scene, with such commentary observed from streamers Moppski, Deki1, and Mr_nubi on June 15, 2019 as one instance of many). But art streamers also occasionally reflect upon subject, technique, and practice in ways that explore both the creation of the work itself as well as the performative act of creating work for a television program (which is related in obvious fashion to particular aspects of streaming).

The individual forms of work involved in streaming are numerous. Art streamers that were observed as a part of this work experimented with multiple cameras and technologies in order provide views of 1) the work being created (either digital -i.e. screenshare, or physical -i.e. webcam), 2) their hands 
using the brush, stylus, tablet surface, or other input device during the physical act of painting or drawing, 3) their face or other representation such that a live avatar was presented for narration, persona, and engagement, 4) views of the software menus, palettes, and layers (if digital), and/or materials, palette, and studio space (if physical). This is in addition to, or forms a layer of complexity on top of, the multiple forms of engagement required by streamers to engage technically with the platform in effective fashion, which include camera management, music, sound, the augmentation of their streams with bots, gifs, surveys, and more. In this manner, the art streamers that were observed were at once needing to engage deeply with the creation of their individual works, and simultaneously balance that labor against the work of multiple technical activities in order to effectively stream the activity in question such that it could be well understood by their audience. This is illustrated in Figure 1, a screenshot of streamer Taudriel engaged in watercolor work, with a stream cam showing her seated in a gaming chair, a second camera showing her workspace and hand movements, a chat in which she is derided for not answering her Instagram messages, and more.

This form of additional streaming management, in combination with the creation and narration of the work itself, is intended to elicit feedback from likeminded art streamers and critics alike. This involves the work of seeking and cultivating an audience, negotiating feedback cycles and chat monitoring while also working live, learning to give critique to others and to make effective use of feedback, and navigating when to take feedback during the creation of work all processes similar to game and game development streamers $[13,14]$ as previously presented. Yet we witnessed multiple art streamers attempting to impose additional rules on their Twitch-chat or Discord to limit feedback on their work to certain times or modalities, such that the audience was giving more feedback at highly structured times (i.e. once a given area was completed or once a design decision was being considered) but not others (i.e. when a particular area was in development). In this manner, the idea of Bank's 'co-creative' activity [3] as well as Jenkins' participatory culture [21] still holds, but is moderated and structured through the labor of the streamer and the demands of art making (rather than gameplay rhythms) in addition to that of the audience.

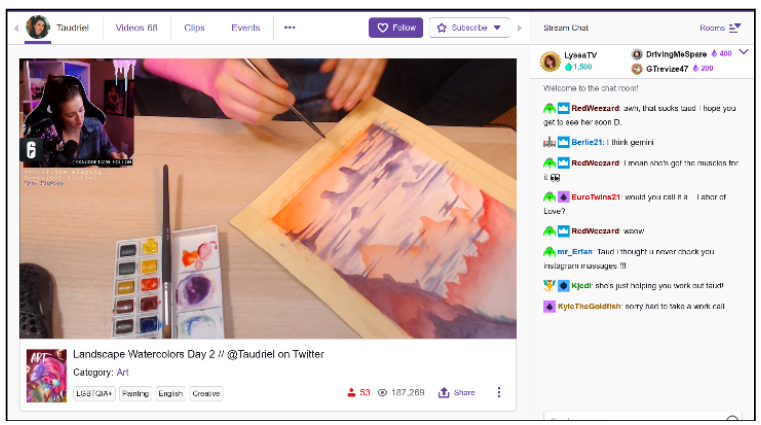

Figure 1. Streamer Taudriel engaged in watercolor work, with associated stream cam, chat, and other elements of the Twitch platform.

Unlike more technically oriented development streams, some art streamers are increasingly focused on evaluating their work in formal terms, similar to O'Donnell's description of 'game talk' [29] and relating their work to both current commercial successes as well as classical works that are culturally recognized. There are additional areas of interest around cultural crossover as streams look to create dual-language streams for cross-over forms and formats, anime and manga drawing being two such examples (one such example is the streamer 'miso_soup333' and their paint sessions typically labelled 'ENG/JP' denoting a stream in English but with some Japanese language). Additionally, the practice of these streamers often involves the prenegotiation of 'attend my stream and I'll attend yours' for both collaboration and review, as well as the bolstering of Twitch-based metrics (observed in stream chat Jan 14, 2019). Individual streams and groups arise from common artistic practices (i.e. exploring 'brushing technique' or 'experimenting with palette' or particular forms or subjects), as well as process-oriented paradigms such as 'sketch to paint' or 'polishing and print techniques', as observed in a series of selected streams on Dec 10,12, 15, and 16 in 2018 and Jan 4 2019, respectively.

This leads to an emergent focus on informal art education within these channels, in ways that engage participants directly in the labor of education, with similar forms and themes relative to the prior discussion of games, play, and labor surrounding mentorship and the creation of guides and walkthroughs, leveling services, etc. Some of the art streamers in this study had created tutorials for various techniques or practices, while others themed various streams or sessions as a form of "watch me recreate a more famous work' or 'watch me illustrate a particular character'. Still others were known to various members of their community for 'giving good feedback' or 'providing good resources' as well as being talented artists and producing meaningful work 
themselves, and in this sense the work of engaging in meaningful critique in combination with demonstrated practice was a way of accruing a sort of 'art streamer capital' similar to the notion of 'gaming capital' as discussed by Consalvo [10]. It implies sustained labor in both engagement with the community, with personal work, with informal contributions to the learning of others, and participation in the entire scene in accordance with the norms and values of the community.

Yet in streaming, the scale and format of critique in a given online space often matures organically from a somewhat sophomoric form to discussion of both art streaming as a performative act as well as a discussion of the piece and critique of artistic process. This has similarities to work using weblogs and other forms for reflection [5,30]. There is also an informal system of skill levelling and knowledge acquisition as individual streamers seek to associate with others who are producing work at a similar or slightly aspirational level, in addition to pursuit of consuming celebrity streams or post-mortems of famous work.

\subsection{Monetization}

Some streamers are also clearly motivated to move beyond the pure-hobby aspect of art streaming and attempt to commercialize their activity to varying degrees, much like the YouTubers detailed by Postigo [32]. Of the streamers observed in this work, this was actually a relatively small subset (approximately $1 / 5^{\text {th }}$ of the streams observed had taken noticeable steps to incorporate tools or platforms to attempt to monetize their activity). The labor here again takes various forms that range from engaging with technological elements of the platform to sustained marketing and social engagement. Streamers that are seeking donations in support of their work are often using a third-party platform for such, of which Patreon is the most popular, particularly given its membership-based model for recurring support. PayPal was also a common choice for one-time donations.

Art streamers would at times integrate bots to remind channel participants for donations and support, as well as integrated tiles and buttons for such on their Twitch page. Here we see again how the Twitch logics originally created for game streaming are repurposed for art streamers. Yet the art streamers have needs that go beyond listing social media channels or a tips jar. We noted that it was also common among this community for artists to sell completed work, and many hosted galleries on various social media channels (Instagram, etc.) as well as specialized websites and services catering to creative media such as Deviant Art [47] and Adobe BeHance [48].
Occasionally various pieces would be listed for sale on the Twitch page using a custom widget, but more likely artists would maintain a separate website or gallery listing such offerings. Thus the labor practices here are intense as they require maintaining a social media presence, an online gallery, channels for donations, billing, and financial transactions, and ensuring the linkages between all of these pieces are current, well maintained, and obvious to a potential audience, all of which is on top of the work of actually creating art - i.e. the core concept of engagement in the activity. This is shown in Figure 2, a screenshot of the various channels, outlets, galleries, and communication channels for streamer BlackKurone, which are typical of a 'freelance' approach to art streaming. Very few if any of the streamers observed were demonstrably receiving donations at a scale that would support even a basic income (note BlackKurone's note that 'donations will be for living for me and my doggo') - there does not yet appear to be a substantial 'celebrity artists stream' or 'artSports' style phenomenon that has integrated advertising dollars and associated scale, although again given the Ross-style crossovers this is certainly a long term possibility as Twitch continues to engage with both its 'Creative' brand and popular culture.

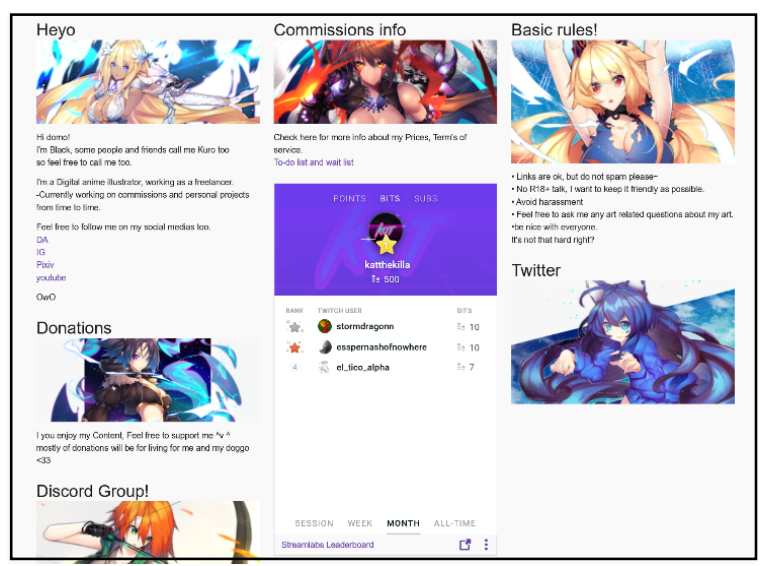

Figure 2: Screenshot of the various galleries, websites, channels, and communications for art streamer BlackKurone

\subsection{Community and engagement}

The work of streamers to cultivate and grow their communities both in terms of platforms for engagement, co-creation, and feedback, as well as a potential commercial audience, is endless, and similar to the "relational labor" that Baym has found musicians must now engage in to have successful commercial careers [4]. Far from being limited to the 'Twitch Art' channel, streamers in this space are likely to engage in multiple areas of streaming culture. There 
is significant anecdotal evidence that these linkages, relationships, and connection patterns are informed by, and intertwined with, other networks that surround commercial games and esports: members of a given league or team that share a practice and affinity for game art creation seek to connect in ways that are semi-exclusionary from the rest of their team or guild, yet are also seeking additional membership and engagement from other practitioners through these networks. Thus, the streaming platform, and its connection to esports, professionalized gaming, casual game play, and other activities is not formally divided but rather forms a mosaic of connected experiences that streamers and audience members dip across depending on interests, focus, and availability. Art streamers are constantly engaged in the work of encouraging potential viewers, seeking potential collaborators, and growing an audience of potential patrons, regardless of what else they might be engaged in.

\subsection{Toxicity}

Finally, some streamers are forcibly engaged in the labor of mitigating or managing toxicity, mainly due to being a woman, person of color, or recognizably LGBTQ + . It should come as no surprise in the postGamergate world $[7,19,33]$ that this type of harassment and discrimination would be present, and it is consistent with more generalized work on streaming in general $[8,38,41]$. At first pass, the streams observed in this study seemed to have a lower incidence of such behavior than larger more gamefocused streams, and there was often a very open, supportive, and inclusive operational practice (as is common amongst the creative community on Twitch). Sometimes though there remained a fixation on sexualized subject matter coupled with observed instances of individual streamers attempting to exert 'expert knowledge' (which was sometimes academically problematic) through either formalism (i.e. terminology) and/or raw skill (i.e. early mastery of technique) to dominate the conversation and discourse (observed in stream on Jan 27, 2019, as one instance among many). In almost all of these cases we observed, the subject matter of predominantly white men was never challenged as to its content or even the depiction of sexualized subject matter, while that of women, persons of color, or streamers who identify as LGBTQ+ (or present themselves in a manner that such might be assumed) are likely to face additional scrutiny. In these instances, members of the audience would begin commenting not on the artwork but on the personal appearance or mannerisms of the streamer, with typical toxicity and gendered biases consistent with larger trends in gaming culture. This in turn necessitates both the emotional labor of dealing with and responding to such activity, as well as the technical labor surrounding chat monitoring, reporting, banning, and securing accounts and links against further harassment. Twitch itself as a platform has proven largely disinterested in proactively assisting streamers in such cases, or in dealing effectively with the culture writ large.

\subsection{Authenticity in art streaming}

All of these various forms of labor are enmeshed and intertwined in a complex ecosystem that positions the work of art streaming towards a new idealism of authenticity. By engaging in this work, the overall aesthetic of the art streamer is not merely an educational construct, an exercise in entertainment, or a kind of symbiotic completionism. Rather, art streaming opens the process of art creation for view, and it is this fact that resonates with regard to educational potential, but also commercial exploitation. The idea of custom works, of an authenticity informed not just by a uniqueness in the final product but by the nature and process of how that work was made is an evolution in the public gestalt in the age of online ordering and near-instant gratification. This parallels the discussion in production circles about mass customization $[36,43]$ which articulates that in the era following mass production value is derived from the ability to customize products such that they feel unique and specialized to the owner. It also bears similarity to the now-common practice of posting 'in progress' photographs or screenshots for everything from architecture to video games to woodworking as an audience engagement tactic. Patrons taking commissions from these streaming artists not only get a custom work, they are able to observe the creation of the work in real-time in ways that echo the live public painting of murals, schools, churches, and other public spaces. The performance of the process itself becomes a part of the work, in which the patron can engage directly to whatever extent agreeable between the parties in question, noting, of course, the financial power advantage in that negotiation of labor. In this manner the commissioning of such work not only purchases the end product, it purchases the process by which it is created, and in this it prefigures much of what is now emerging in the 'gig economy'. While this is relatively infrequent in the current practice on Twitch given the relative paucity of financial support for the majority of art streamers, this potentially prefigures an emerging focus on process and engagement on larger economic platforms such as 
Etsy and Pinterest, which are beginning to define the value of items via uniqueness.

\section{Conclusion}

Throughout this work, numerous parallels and similarities regarding play and labor have been illustrated that extend first from the labor of playing games themselves into the labor of streaming live gameplay, and from there extended yet further into game-adjacent spaces such as art streaming. Play and labour are not opposed and are in fact intermingled in these activities, in ways that are becoming highly platformized. These platforms, because they are treating individualized content generically, are extending the labor conditions and practices surrounding one activity (gameplay) into another (art streaming), and in fact form a basis for certain types of labor conditions in and of themselves. Despite this, individual communities are constantly exploring various modifications, sub-culture practices, and extensible norms and values to build on top of these platforms practices and techniques that address some of these conditions. This has potentially significant impact when examined from the viewpoint of larger socio-economic trends, and as such the work of examining games, streaming, and culture is critical to that larger discourse.

This initial analysis of our data suggests that significant and varied labor is being performed as part of the process of live streaming, whether the subject matter is game playing or making art, and that such practices are strikingly similar across game and nongame streams. Our future efforts involve investigating how such practices might change over time including both trends within/across different streaming activities, as well as the evolving practices of individual streamers. The large number of streams we investigated suggest a certain consistency is already developing, yet further investigation particularly of higher profile art streamers - should be done to determine if there are significant differences. Finally, different live streaming platforms should be studied comparatively, to see if Twitch is still the locus for live streaming in North America, or if interesting and important differences are emerging elsewhere.

\section{References}

[1] Julia Alexander. 2018. Twitch is ditching IRL label, introducing distinct categories for ASMR, vlogging and more. Polygon. Retrieved June 15, 2019 from https://www.polygon.com/2018/8/10/17674306/twitch-irlcreative-new-categories
[2] Ross J. Anderson. 2010. Security Engineering: A Guide to Building Dependable Distributed Systems. John Wiley \& Sons.

[3] John Banks. 2015. Co-creating Videogames. Bloomsbury.

[4] Nancy K. Baym. 2018. Playing to the Crowd: Musicians, Audiences, and the Intimate Work of Connection. NYU Press.

[5] Diane M. Bender and Jon D. Vredevoogd. 2006. Using Online Education Technologies to Support Studio Instruction. Journal of Educational Technology \& Society 9, 4 (2006), 114-122.

[6] Tom Boellstorff. 2008. Coming of age in Second Life: an anthropologist explores the virtually human. Princeton University Press, Princeton.

[7] Andrea Braithwaite. 2016. It's About Ethics in Games Journalism? Gamergaters and Geek Masculinity. Social Media + Society 2, 4 (November 2016), 205630511667248.

DOI:https://doi.org/10.1177/2056305116672484

[8] Mia Consalvo. forthcoming. Kaceytron and transgressive play on Twitch.tv. In Transgressions in games and play, Kristine Jørgensen and Faltin Karlsen (eds.). MIT Press, Cambridge, MA.

[9] Mia Consalvo. 2003. Zelda 64 and Video Game Fans: A Walkthrough of Games, Intertextuality, and Narrative. Television \& New Media 4, 3 (August 2003), 321-334. DOI:https://doi.org/10.1177/1527476403253993 [10] Mia Consalvo. 2007. Cheating: gaining advantage in videogames. MIT Press, Cambridge, Mass.

[11] Mia Consalvo. 2016. Atari to Zelda: Japan's videogames in global contexts. MIT Press, Cambridge, MA.

[12] Mia Consalvo. 2019. Gaming Capital and Clash Royale. In How to Play Video Games, Matthew Payne and Nina Huntemann (eds.). NYU Press, New York, NY, 185192.

[13] Mia Consalvo and Phelps, Andrew. 2019. Performing Game Development Live on Twitch. In Proceedings of the 52nd Hawaiian International Conference on Systems Science.

[14] Consalvo, Mia and Marilyn Sugiarto. 2016. Game Over? Not Really: Spectating Failure on Twitch.tv.

[15] Jie Deng, Felix Cuadrado, Gareth Tyson, and Steve Uhlig. 2015. Behind the game: Exploring the twitch streaming platform. In 2015 International Workshop on Network and System Support for Games (NetGames). DOI:https://doi.org/DOI:http://

dx.doi.org/10.1109/NetGames.2015.7382994

[16] Julian Dibbell. 2007. The Life of a Chinese Gold Farmer. The New York Times. Retrieved June 15, 2019 from https://www.nytimes.com/2007/06/17/magazine/17lootfarm ers-t.html

[17] Nick Dyer-Witheford and Greig De Peuter. 2009. Games of Empire: Global Capitalism and Video Games. U of Minnesota Press, Minneapolis.

[18] Tarleton Gillespie. 2010. The politics of 'platforms.' New Media \& Society 12, 3 (May 2010), 347-364. DOI:https://doi.org/10.1177/1461444809342738 
[19] Kishonna L. Gray, Bertan Buyukozturk, and Zachary G. Hill. 2017. Blurring the boundaries: Using Gamergate to examine "real" and symbolic violence against women in contemporary gaming culture. Sociology Compass 11, 3 (March 2017), e12458.

DOI:https://doi.org/10.1111/soc4.12458

[20] William A. Hamilton, Oliver Garretson, and Andruid Kerne. 2014. Streaming on twitch: fostering participatory communities of play within live mixed media. 1315-1324. DOI:https://doi.org/10.1145/2556288.2557048 [21] Henry Jenkins. 2008. Convergence Culture. NYU Press, New York, NY. Retrieved June 15, 2018 from http://nyupress.org/books/9780814742952/

[22] Mark R. Johnson and Jamie Woodcock. 2017. 'It's like the gold rush': the lives and careers of professional video game streamers on Twitch.tv. Information, Communication \& Society (October 2017), 1-16. DOI:https://doi.org/10.1080/1369118X.2017.1386229 [23] Jesper Juul. 2010. A casual revolution: reinventing video games and their players. MIT Press, Cambridge, MA.

[24] Julian Kücklich. 2005. FCJ-025 Precarious Playbour: Modders and the Digital Games Industry. 5 (2005). Retrieved June 15, 2019 from

http://five.fibreculturejournal.org/fcj-025-precariousplaybour-modders-and-the-digital-games-industry/ [25] Ian MacInnes. 2004. The Implications of Property Rights in Virtual Worlds. New York (2004), 8.

[26] Thomas Malaby. 2007. Beyond play: A new approach to games. Games \& Culture 2, 2 (2007), 95-113. [27] Joe Marino. 2017. Trying To "Make It" As A Twitch Streamer Could Have Killed Me. Kotaku. Retrieved June 15, 2019 from https://kotaku.com/trying-to-make-itas-a-twitch-streamer-almost-killed-me-1792711102 [28] Lisa Nakamura. 2009. Don't Hate the Player, Hate the Game: The Racialization of Labor in World of Warcraft. Critical Studies in Media Communication 26, 2 (June 2009), 128-144.

DOI:https://doi.org/10.1080/15295030902860252

[29] Casey O'Donnell. 2014. Developer's Dilemma: The Secret World of Videogame Creators. MIT Press.

[30] Alexandra Overby. 2009. The New Conversation: Using Weblogs for Reflective Practice in the Studio Art Classroom. Art Education 62, 4 (July 2009), 18-24. DOI:https://doi.org/10.1080/00043125.2009.11519025 [31] H. Postigo. 2008. Video Game Appropriation through Modifications: Attitudes Concerning Intellectual Property among Modders and Fans. Convergence: The International Journal of Research into New Media Technologies 14, 1 (February 2008), 59-74. DOI:https://doi.org/10.1177/1354856507084419 [32] Hector Postigo. 2016. The socio-technical architecture of digital labor: Converting play into YouTube money. New Media \& Society 18, 2 (2016), 332-349.

[33] Anastasia Salter and Bridget Blodgett. 2017. Toxic Geek Masculinity in Media. Springer International Publishing, Cham. DOI:https://doi.org/10.1007/978-3-31966077-6

[34] Tanja Sihvonen. 2009. Players unleashed! Modding The Sims and the culture of gaming. Retrieved January 20 , 2017 from http://citeseerx.ist.psu.edu/viewdoc/download?doi=10.1.1.9 $21.4343 \&$ rep $=$ rep $1 \&$ type $=$ pdf

[35] Olli Sotamaa. 2010. When the Game Is Not Enough: Motivations and Practices Among Computer Game Modding Culture. Games and Culture 5, 3 (July 2010), 239-255. DOI:https://doi.org/10.1177/1555412009359765 [36] Carsten Svensson and Ari Barfod. 2002. Limits and opportunities in mass customization for "build to order" SMEs. Computers in Industry 49, 1 (September 2002), 7789. DOI:https://doi.org/10.1016/S0166-3615(02)00060-X [37] T. L. Taylor. 2006. Does WoW Change Everything?: How a PvP Server, Multinational Player Base, and Surveillance Mod Scene Caused Me Pause. Games and Culture 1, 4 (October 2006), 318-337.

DOI:https://doi.org/10.1177/1555412006292615

[38] T. L. Taylor. 2018. Watch me play: twitch and the rise of game live streaming. Princeton University Press, Princeton, NJ.

[39] Dorothy E Warner and Mike Raiter. 2005. Social Context in Massively-Multiplayer Online Games (MMOGs): Ethical Questions in Shared Space. International Review of Information Ethics 4, (2005), 7. [40] Nicholas Watson. 2019. Re-Crafting Games: The Inner Life of Minecraft Modding. Concordia University, Montreal, Canada.

[41] Donghee Yvette Wohn. 2019. Volunteer Moderators in Twitch Micro Communities: How They Get Involved, the Roles They Play, and the Emotional Labor They Experience. In Proceedings of the 2019 CHI Conference on Human Factors in Computing Systems - CHI '19, 1-13. DOI:https://doi.org/10.1145/3290605.3300390

[42] Nick Yee. 2006. Yi-Shan-Guan. The Daedalus Project. Retrieved June 15, 2019 from

http://www.nickyee.com/daedalus/archives/print/001493.ph $\mathrm{p}$

[43] 2014. Have it Your Way: Manufacturing in the Age of Mass Customization. IndustryWeek. Retrieved June 15, 2019 from

https://www.industryweek.com/technology/have-it-yourway-manufacturing-age-mass-customization

[44] The Joy of Painting. Retrieved June 15, 2019 from http://www.imdb.com/title/tt0383795/

[45] Bob Ross - Weekend Marathon. Bob Ross Weekend Marathon. Retrieved June 15, 2019 from https://www.twitch.tv/bobross

[46] Cabin chance. Retrieved June 15, 2019 from https://www.facebook.com/cabinchance/

[47] DeviantArt - Discover The Largest Online Art Gallery and Community. DeviantArt. Retrieved June 15, 2019 from https://www.deviantart.com/

[48] Behance. Retrieved June 15, 2019 from https://www.behance.net/ 